\title{
Impact of Axillary Dissection Among Patients With Sentinel Node-Positive Breast Cancer Undergoing Mastectomy
}

\author{
James Sun, MD'; Brittany J. Mathias, MD,2; Christine Laronga, MD'; Weihong Sun, MD; Jun-Min Zhou, PhD3;
} William J. Fulp, MS ${ }^{3,4}$; John V. Kiluk, MD ${ }^{1}$; and M. Catherine Lee, MD ${ }^{1}$

\section{ABSTRACT}

Background: Results of the American College of Surgeons Oncology Group (ACOSOG) Z0011 trial supports omission of completion axillary lymph node dissection (CLND) after breast-conservation surgery with a positive sentinel lymph node biopsy (SLNB). We hypothesized that CLND also does not impact outcomes in women with clinically node-negative (cNO), pathologically node-positive breast cancer undergoing mastectomy. Materials and Methods: A single-institution retrospective review was performed of patients with SLN-positive breast cancer treated from July 1999 through May 2018. Clinicopathologic and outcome data were collected. Patients with SLNBs were compared with those receiving SLNB and CLND. The KruskalWallis, chi-square, and Fisher exact tests were used to assess for differences between continuous and categorical variables. The logrank test was used for time-to-event analyses, and Cox proportional hazards models were fit for locoregional and distant recurrence and overall survival (OS). Results: Of 329 patients with SLN-positive breast cancer undergoing mastectomy, 60\% had CLND $(n=201)$. Median age at diagnosis was 53 years (interquartile range [IQR], 46-62 years). The median number of SLNs sampled was 3 (IQR, 2-4), and the median number of positive SLNs was 1 (IQR, 1-2). Patients receiving CLND had higher tumor grades $(P=.02)$ and a higher proportion of hormone receptor negativity (estrogen receptor, $19 \%$; progesterone receptor, $27 \%$; both $P=.007$ ). A total of 44 patients (22\%) had increased $N$ stage after CLND. Median follow-up was 51 months (IQR, 29-83 months). No association was found between CLND and change in OS and locoregional or distant recurrence. Completion of postmastectomy radiotherapy was associated with improved OS $(P=.04)$. Conclusions: CLND is not significantly correlated with reduced recurrence or improved OS among patients who have $\mathrm{CNO}$, SLN-positive breast cancer treated with mastectomy. CLND was significantly correlated with receipt of adjuvant systemic therapy. Completion of postmastectomy radiotherapy was associated with improved OS.

J Natl Compr Canc Netw 2021;19(1):40-47 doi: $10.6004 /$ jnccn.2020.7597

\footnotetext{
${ }^{1}$ Department of Breast Oncology, Moffitt Cancer Center, Tampa, Florida; ${ }^{2}$ Now with Mercy Clinic Breast Surgery - Coletta, Oklahoma City, Oklahoma; ${ }^{3}$ Department of Biostatistics and Bioinformatics, Moffitt Cancer Center, Tampa, Florida; and ${ }^{4}$ Now with Fred Hutchinson Cancer Research Center, Seattle, Washington.
}

\section{Background}

Surgical management of invasive breast cancer has moved toward less-invasive measures, including axillary management. Axillary lymph node dissection is associated with significant morbidity and complications (wound infections, seromas, paresthesias, and lymphedema) ${ }^{1,2}$ Currently, there is sufficient evidence to support omission of completion axillary lymph node dissection (CLND) in patients undergoing breast-conserving surgery (BCS) with micrometastatic or macrometastatic disease in up to 2 sentinel lymph nodes (SLNs). Both the American College of Surgeons Oncology Group (ACOSOG) Z0011 ${ }^{3}$ and IBCSB 23-01 trials ${ }^{4}$ evaluated patients with earlystage breast cancer who had SLN micrometastases and randomized them to CLND versus observation, reporting no differences in outcomes. ${ }^{5-7}$ In both of these highimpact trials, most patients received some form of adjuvant systemic therapy and/or radiotherapy (RT).

To study the effect of axillary RT, the AMAROS trial assessed whether it provided regional control comparable to that of CLND with fewer adverse effects. ${ }^{8}$ At median 10-year follow-up, no significant differences were seen in overall survival (OS) and locoregional recurrence. ${ }^{9}$ In addition, axillary RT resulted in significantly less morbidity, including lymphedema. Notably, most patients had T1N1 disease, and only $18 \%$ received mastectomy.

These studies, among several others, form the basis for de-escalation of axillary surgery in early-stage breast cancer after BCS, which is the recommended surgical treatment of unifocal, early-stage breast cancer. Although there is mounting evidence that CLND can be omitted in patients who undergo mastectomy, the NCCN Clinical Practice Guidelines in Oncology (NCCN Guidelines) for Breast Cancer state that this should be considered in patients with micrometastatic nodal disease and only if they meet all ACOSOG Z0011 criteria (T1/2 tumors, 1-2

\section{See JNCCN.org for supplemental online content.}


positive SLNs, primary BCS, planned whole-breast RT, and no preoperative chemotherapy). ${ }^{10}$ The American Society of Breast Surgeons consensus statement for axillary management further specifies that the positive SLNs must not have gross extracapsular extension and that patients should complete appropriate adjuvant systemic therapy. ${ }^{11}$

We hypothesized that CLND does not significantly change recurrence rates or OS in women with cN0 breast cancer found to have $\leq 3$ positive lymph nodes after SLNB treated with mastectomy. The goal of this study was to report outcomes of patients who underwent mastectomy with and without CLND for SLN-positive breast cancer. We also analyzed patients who completed postmastectomy RT (PMRT) to determine its contribution to outcomes.

\section{Materials and Methods}

\section{Patient Selection}

After obtaining approval from the University of South Florida Institutional Review Board, we performed a single-institution retrospective review of female patients with their first diagnosis of invasive breast cancer in July 1, 1999, through May 31, 2018. We selected patients who were cN0, SLN-positive (pN1mi-pN3), without distant metastasis and who underwent total or skin-sparing mastectomy and SLNB with or without CLND. Patients with isolated tumor cells [pNO(1+)], those who received preoperative (neoadjuvant) systemic chemotherapy, and those without records of adjuvant therapy were excluded. Patients were identified from a prospectively maintained breast cancer database at an NCI-designated Comprehensive Cancer Center. Clinicopathologic variables, including demographic information, pathology, treatment, recurrence, and survival data, were collected from the medical record. Given the time span from which patients were included, all tumors were restaged according to the 7th edition of the AJCC Cancer Staging Manual. Consent was obtained from most patients for SLNB, intraoperative evaluation of SLN, and CLND if nodes were positive, whereas a smaller subset of patients was brought back to the operating room at a later date for CLND. Patients were discussed in a multidisciplinary conference as needed.

\section{Statistical Analysis}

Patients who received SLNB alone were compared with those who received SLNB and CLND. This analysis was repeated for patients with 3 groups of receptor profiles: estrogen receptor (ER)-positive, HER2-overexpressing, and triple-negative tumors. We then performed a subset analysis by completion of PMRT. Kruskal-Wallis tests were used to assess differences between continuous variables, and chi-square and Fisher exact tests were used to evaluate differences between categorical variables. Time to locoregional recurrence, distant recurrence, and OS were compared using the log-rank test. Univariate Cox proportional hazards models were fit for locoregional recurrence, distant recurrence, and OS. Time-to-event analyses were calculated from the date of surgery. $P$ values were not adjusted for multiple comparisons. A $P$ value $<.05$ was considered statistically significant. All analyses were performed using $\mathrm{R}$ version 3.5.0 and 3.5.1 (R Foundation for Statistical Computing).

\section{Results}

We identified 340 female patients with cN0, SLN-positive invasive breast cancer without distant metastasis who underwent mastectomy from July 1999 through May 2018. Eleven patients refused adjuvant therapy, leaving 329 patients for analysis (Figure 1). Median age at diagnosis was 53 years (interquartile range [IQR], 46-63 years), 303 patients were White (91\%), and median body mass index was $26.4 \mathrm{~kg} / \mathrm{m}^{2}$ (IQR, $23-30 \mathrm{~kg} / \mathrm{m}^{2}$ ). A total of 136 patients $(41 \%)$ underwent surgery before September 2010, the month in which ACOSOG Z0011 was published. ${ }^{3}$

Most tumors were unifocal (74\%), T2 (48\%), with a median size of $2.3 \mathrm{~cm}$ (IQR, $1.5-3.5 \mathrm{~cm}$ ), and were invasive ductal subtype $(73 \%)$, grade $2(47 \%)$, without lymphovascular invasion (57\%), ER-positive (85\%), progesterone receptor (PR)-positive (78\%), and without

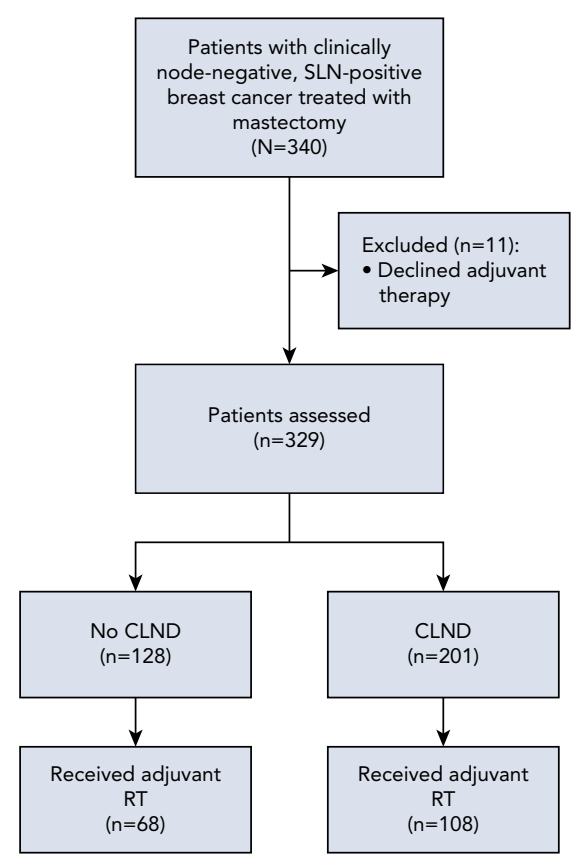

Figure 1. CONSORT diagram of patients in the study cohort. Abbreviations: CLND, completion lymph node dissection; RT, radiotherapy; $\mathrm{SLN}$, sentinel lymph node. 


\begin{tabular}{|c|c|}
\hline Characteristic & n (\%) \\
\hline Total, N & 329 \\
\hline Median age at diagnosis (IQR), y & $53(46-63)$ \\
\hline Median BMI (IQR), kg/m² & $26.4(23-30)$ \\
\hline Median tumor size (IQR), cm & $2.3(1.5-3.5)$ \\
\hline \multicolumn{2}{|l|}{ Histology } \\
\hline IDC & $241(73)$ \\
\hline ILC & $54(16)$ \\
\hline IMC & $12(4)$ \\
\hline Other & $22(7)$ \\
\hline \multicolumn{2}{|l|}{ Tumor grade ${ }^{a}$} \\
\hline 1 & $38(12)$ \\
\hline 2 & $153(47)$ \\
\hline 3 & $136(41)$ \\
\hline \multicolumn{2}{|l|}{ Pathologic T stage } \\
\hline 1 & $144(44)$ \\
\hline 2 & $159(48)$ \\
\hline 3 & $26(8)$ \\
\hline \multicolumn{2}{|l|}{ Overall stage } \\
\hline 1 & $44(13.5)$ \\
\hline II & $244(74)$ \\
\hline III & $41(12.5)$ \\
\hline \multicolumn{2}{|l|}{ Estrogen receptor } \\
\hline Negative & $49(15)$ \\
\hline Positive & $280(85)$ \\
\hline \multicolumn{2}{|l|}{ Progesterone receptor } \\
\hline Negative & $73(22)$ \\
\hline Positive & $256(78)$ \\
\hline \multicolumn{2}{|l|}{ HER2 ${ }^{a}$} \\
\hline Negative & 291 (89) \\
\hline Positive & $37(11)$ \\
\hline
\end{tabular}

HER2 overexpression (89\%). The median number of SLNs removed was 3 (IQR, 2-4), and the median number of positive SLNs was 1 (IQR, 1-2). A total of 201 patients underwent CLND (61\%), and the median number of nodes obtained was 15 (IQR, 11-19) (Table 1). Of the patients who underwent CLND, 85 (42\%) had additional nodal disease.

In terms of adjuvant therapy, 176 (53\%) of 329 patients received RT, 245 (75\%) received chemotherapy, and $272(83 \%)$ received endocrine therapy (Table 1$)$. Records of systemic therapy regimens, sites of adjuvant $\mathrm{RT}$, and radiation doses were not available. Over a median follow-up of 51 months (IQR, 29-83 months),

\begin{tabular}{|c|c|}
\hline Characteristic & n (\%) \\
\hline \multicolumn{2}{|l|}{ Surgery type } \\
\hline TM & $208(63)$ \\
\hline SSM & $121(37)$ \\
\hline Median SLNs removed (IQR), n & $3(2-4)$ \\
\hline Median positive SLNs (IQR), n & $1(1-2)$ \\
\hline \multicolumn{2}{|l|}{ Extracapsular extension } \\
\hline Yes & $104(32)$ \\
\hline No & $225(68)$ \\
\hline \multicolumn{2}{|l|}{ CLND } \\
\hline Yes & $201(61)$ \\
\hline No & $128(39)$ \\
\hline \multicolumn{2}{|l|}{ Surgery timing ${ }^{b}$} \\
\hline Before September 2010 & $136(41)$ \\
\hline After September 2010 & $193(59)$ \\
\hline \multicolumn{2}{|l|}{ Treated with adjuvant RT } \\
\hline Yes & $176(53)$ \\
\hline No & $153(47)$ \\
\hline \multicolumn{2}{|c|}{ Treated with adjuvant chemotherapy } \\
\hline Yes & $245(75)$ \\
\hline No & $84(25)$ \\
\hline
\end{tabular}

Abbreviations: BMI, body mass index; CLND, completion axillary lymph node dissection; IDC, invasive ductal carcinoma; ILC, invasive lobular carcinoma; IMC, invasive mammary carcinoma; IQR, interquartile range; $R T$, radiotherapy; SLN, sentinel lymph node; SSM, skin-sparing mastectomy; TM, total mastectomy.

aData not available for all patients.

bDate of American College of Surgeons Oncology Group Z0011 publication.

47 patients (14\%) had disease recurrence, 9 (3\%) had locoregional recurrences, and $38(12 \%)$ had distant recurrences.

\section{Comparison by CLND}

The 201 patients who underwent mastectomy and CLND were significantly younger (median age, 51 years [IQR, $43-61$ years] vs 56 years [IQR, $47-64$ years]; $P=.003$ ) and tended to have more aggressive, higher-stage disease $(P<.001)$, with a higher proportion of high-grade tumors $(P=.02)$ with lymphovascular invasion $(46 \%$ vs $31 \% ; P=.01)$. These tumors tended to be ER-negative ( $19 \%$ vs $8 \% ; P=.007)$ and PR-negative $(27 \%$ vs $14 \%$; $P=.007)$, although HER2 expression was not significantly different $(P=.17)$ (Table 2$)$.

We attempted to further evaluate factors that affect outcome after CLND or SLNB only. Among the 3 subtypes analyzed, the HER2-negative and triple-negative analyses were limited by low sample size. However, among patients with ER-positive breast cancer $(n=280)$, we found patterns 


\begin{tabular}{|c|c|c|c|}
\hline Characteristic & No CLND n (\%) & CLND n (\%) & $P$ Value $^{a}$ \\
\hline Total & $128(39)$ & $201(61)$ & \\
\hline $\begin{array}{l}\text { Median age at diagnosis } \\
\text { (IQR), y }\end{array}$ & $56(47-64)$ & $51(43-61)$ & .003 \\
\hline Median BMI (IQR), kg/m² & $26.1(23.3-30.9)$ & $26.4(22.9-29.7)$ & .52 \\
\hline $\begin{array}{l}\text { Median tumor size (IQR), } \\
\mathrm{cm}\end{array}$ & $2.1(1.48-3.0)$ & $2.5(1.7-3.5)$ & .02 \\
\hline Histology & & & .75 \\
\hline IDC & $94(74)$ & $147(73)$ & \\
\hline ILC & $23(18)$ & $31(16)$ & \\
\hline $\mathrm{IMC}$ & $3(2)$ & $9(4)$ & \\
\hline Other & $8(6)$ & $14(7)$ & \\
\hline Tumor grade ${ }^{b}$ & & & .02 \\
\hline 1 & $18(14)$ & $20(10)$ & \\
\hline 2 & $69(54)$ & $84(42)$ & \\
\hline 3 & $41(32)$ & $95(48)$ & \\
\hline Pathologic T stage & & & .34 \\
\hline 1 & $62(48)$ & $82(41)$ & \\
\hline 2 & $58(45)$ & $101(50)$ & \\
\hline 3 & $8(7)$ & $18(9)$ & \\
\hline Overall stage & & & $<.001$ \\
\hline 1 & $32(25)$ & $12(6)$ & \\
\hline ॥ & $88(69)$ & $156(78)$ & \\
\hline III & $8(6)$ & $33(16)$ & \\
\hline Lymphovascular invasion & & & .01 \\
\hline Yes & $40(31)$ & $93(46)$ & \\
\hline No & $86(67)$ & $102(51)$ & \\
\hline Indeterminate & $2(2)$ & $6(3)$ & \\
\hline Estrogen receptor & & & .007 \\
\hline Negative & $10(8)$ & $39(19)$ & \\
\hline Positive & $118(92)$ & $162(81)$ & \\
\hline Progesterone receptor & & & .007 \\
\hline Negative & $18(14)$ & $55(27)$ & \\
\hline Positive & $110(86)$ & $146(73)$ & \\
\hline
\end{tabular}

(continued)

similar to those in the overall cohort; patients undergoing CLND had higher overall stage and were more likely to be treated with adjuvant chemotherapy (both $P<.0001$ ) (supplemental eTable 1, available with this article at JNCCN.org).

After CLND, 44 patients (22\%) had an increase in nodal staging (Table 3). Before CLND, 13 patients had N1mi disease, 27 had N1 disease, and 4 had N2 disease. After CLND, 11 of the patients with N1mi disease were restaged to $\mathrm{N} 1$ and 2 were restaged to $\mathrm{N} 2 ; 13$ of the patients with $\mathrm{N} 1$ disease were restaged to $\mathrm{N} 2$ and 14 were

\begin{tabular}{|c|c|c|c|}
\hline Characteristic & No CLND n (\%) & CLND n (\%) & $P$ Value ${ }^{a}$ \\
\hline HER $2^{b}$ & & & 17 \\
\hline Negative & $117(92)$ & $174(87)$ & \\
\hline Positive & $10(8)$ & $27(13)$ & \\
\hline $\begin{array}{l}\text { Median SLNs removed } \\
\text { (IQR), n }\end{array}$ & $3(2-4)$ & $3(2-4)$ & .80 \\
\hline $\begin{array}{l}\text { Median positive SLNs } \\
\text { (IQR), n }\end{array}$ & $1(1-1)$ & $1(1-2)$ & $<.0001$ \\
\hline Extracapsular extension & & & .02 \\
\hline Yes & $30(23)$ & $74(37)$ & \\
\hline No & 98 (77) & $127(63)$ & \\
\hline Surgery timing ${ }^{c}$ & & & $<.0001$ \\
\hline Before September 2010 & $14(11)$ & $122(61)$ & \\
\hline After September 2010 & $114(89)$ & $79(41)$ & \\
\hline Treated with adjuvant RT & & & 1.0 \\
\hline Yes & $68(53)$ & $108(54)$ & \\
\hline No & $60(47)$ & $93(46)$ & \\
\hline \multicolumn{3}{|c|}{ Treated with adjuvant chemotherapy } & $<.0001$ \\
\hline Yes & $71(55)$ & $174(87)$ & \\
\hline No & $57(45)$ & $27(13)$ & \\
\hline
\end{tabular}

Abbreviations: $\mathrm{BMI}$, body mass index; CLND, completion axillary lymph node dissection; IDC, invasive ductal carcinoma; ILC, invasive lobular carcinoma; $I M C$, invasive mammary carcinoma; IQR, interquartile range; $R T$, radiotherapy; SLN, sentinel lymph node; SSM, skin-sparing mastectomy; TM, total mastectomy.

aBold indicates statistically significant $P$ value.

bData not available for all patients.

'Date of American College of Surgeons Oncology Group Z0011 publication.

restaged to N3; and all of the patients with N2 disease were restaged to N3.

Patients who underwent CLND were more likely to be treated with chemotherapy $(P<.0001)$ but not PMRT. Stratifying by publication date of ACOSOG Z0011 (September 2010), significantly fewer CLNDs $(n=79 ; 41 \%)$ were performed after versus before September 2010 $(P<.0001)$ (Table 2).

In terms of outcomes, CLND was not associated with changes in OS (hazard ratio [HR], 1.61; 95\% CI, 0.65-3.97; $P=.3$ ) or distant recurrence (HR, 1.58; 95\% CI, 0.72-3.5; $P=.26$ ) by univariate analysis, supported by Kaplan-Meier estimates (Figure 2A, B), but it showed a trend toward improved locoregional recurrence (HR, 0.67; 95\% CI, $0.18-2.5 ; P=.55)$. Estimated 5-year survival was $89 \%(95 \%$ CI, $84 \%-94 \%$ ) for patients who received CLND and $93 \%$ (95\% CI, 86\%-99\%) for those who received SLNB alone.

\section{Comparison by Completion of Adjuvant RT}

Patients who received adjuvant RT tended to have a higher proportion of ER-positive and PR-positive tumors 


\begin{tabular}{|c|c|c|}
\hline $\begin{array}{l}\text { Total Patients } \\
\text { Upstaged }\end{array}$ & Pre-CLND & Post-CLND \\
\hline \multirow[t]{3}{*}{44 (22\%) } & $\mathrm{N} 1 \mathrm{mi}, 13$ (6.5\%) & $\begin{array}{l}\mathrm{N} 1,11 \text { of } 13(85 \%) \\
\mathrm{N} 2,2 \text { of } 13(15 \%)\end{array}$ \\
\hline & N1, 27 (84\%) & $\begin{array}{l}\mathrm{N} 2,13 \text { of } 27(48 \%) \\
\mathrm{N} 3,14 \text { of } 27(52 \%)\end{array}$ \\
\hline & N2, 4 (6\%) & $N 3,4$ of $4(100 \%)$ \\
\hline
\end{tabular}

Abbreviation: CLND, completion axillary lymph node dissection.

(both $P=.04$ ) without a significant difference in HER2 expression. They also had higher pathologic $\mathrm{T}$ stage and overall stage and an increase in N stage after CLND (all $P<.0001$ ) (Table 4).

Patients who completed adjuvant RT were more likely to have received chemotherapy $(P<.001)$. Treatment with adjuvant RT showed trends toward improved OS (HR, 0.5 ; 95\% CI, 0.2-1.0; $P=.05$ ) and locoregional control (HR, $0.7 ; 95 \% \mathrm{CI}, 0.2-2.5 ; P=.6$ ) without a decrease in distant recurrence (HR, 1.0; 95\% CI, 0.5-1.9; $P=1.0$ ) (Figure 2C, D).

\section{Discussion}

Axillary management in patients with breast cancer has moved away from more invasive procedures. Many trials have investigated the safety of these changing practices. Based on results from ACOSOG Z0011 and IBCSG 23-01, it is now accepted that CLND can safely be omitted in select patients with limited disease. ${ }^{3,4,7}$ The AMAROS trial showed comparable axillary control with CLND and axillary RT, further supporting the new trend in minimizing more-invasive therapy. ${ }^{8,9}$ Mounting evidence suggests that omission of CLND may not be limited to only patients treated with BCS but also to those undergoing mastectomy. The effects of these important studies are reflected in the management of our patients. Our results show a clear decrease in CLND performed after publication of ACOSOG Z0011, even among patients receiving mastectomy. High-quality evidence is certainly needed to guide the management of these patients.

Our study included patients who underwent mastectomy with a positive SLNB to determine the effect of CLND on outcomes. Our findings show that CLND does not significantly correlate with changes in outcomes among patients with cN0 breast cancer who have undergone mastectomy with a positive SLNB. In our cohort, younger patients with higher-risk disease (higher grade, lymphovascular invasion, extracapsular extension of lymph node metastases, and hormone receptor [ER, PR] negativity) and higher $\mathrm{N}$ and overall stages were more likely to undergo CLND after positive SLNB. These patients were also more likely to have received adjuvant systemic chemotherapy. Our cohort is similar to the patients in ACOSOG Z0011 in terms of age and tumor characteristics. The largest differences are the lower proportion of $\mathrm{T} 1$ disease $(44 \%$ vs $70 \%$ in ACOSOG Z0011), higher proportion of T2 disease ( $48 \%$ vs $30 \%$ in ACOSOG Z0011), and inclusion of T3 disease (8\%), although $\mathrm{T}$ stage was not a significant factor in our analysis.

After a median follow-up of 51 months, we found that CLND did not correlate with changes in locoregional recurrence, distant recurrence, or OS. The estimated 5 -year survival rate was $89 \%$ for patients who received CLND and 93\% for those who received SLNB alone. These figures are comparable with those reported by Giuliano et al. ${ }^{3,12}$ PMRT also did not significantly change locoregional or distant recurrence but was associated with improved OS, although records of nodal irradiation were not available.

We attribute our findings of better survival among patients treated with SLNB alone to the addition of adjuvant RT. Although PMRT unequivocally improved outcomes among patients with $>3$ involved SLNs, recent analyses have found a benefit among patients with T1-2 breast cancer and 1 to 3 involved SLNs. ${ }^{13}$ The data presented in this study and others have led to updated radiation oncology recommendations supporting PMRT in these patients in order to decrease recurrence and breast cancer mortality. ${ }^{14}$ Our practices are in line with these recommendations; we are less likely to treat patients with N1 disease with CLND, because PMRT is beneficial and may account for the survival difference.

Our findings are also consistent with several retrospective studies of patients with breast cancer and nodal disease after SLNB that investigated the necessity of CLND. Milgrom et $\mathrm{al}^{15}$ compared outcomes of patients with positive SLNB treated with mastectomy or BCS and found no significant differences. Notably, the study population had low-risk disease and included No(i+) patients, but they reported only slightly higher regional recurrence rates after exclusion of $\mathrm{NO}(\mathrm{i}+)$ patients. They also reported a low incidence of PMRT among patients treated with mastectomy, and therefore its contribution cannot be accurately assessed based on this study. FitzSullivan et al, ${ }^{16}$ Snow et $a l,{ }^{17}$ and Joo et al ${ }^{18}$ all performed studies comparing small cohorts of patients with SLN-positive breast cancer who underwent mastectomy and were treated with CLND or SLNB alone and found no differences in outcomes. Our study builds on these results with a larger patient cohort.

Several ongoing prospective trials are specifically investigating patients with cN0 SLNB-positive breast cancer treated with mastectomy. The ongoing UK-ANZ POSNOC trial (ClinicalTrials.gov identifier: NCT02401685) seeks to better define the role of axillary treatment in patients undergoing both BCS and mastectomy with 
A

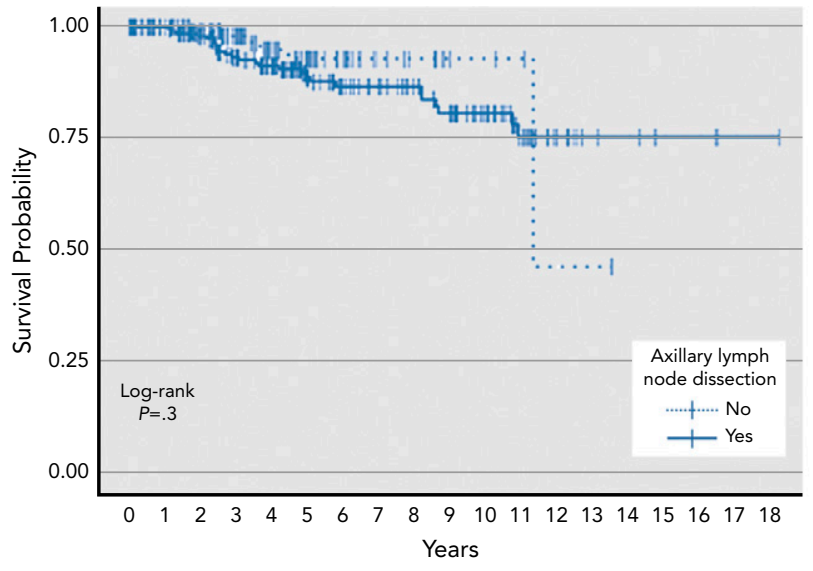
Number at risk

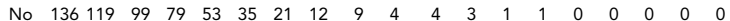

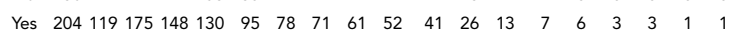

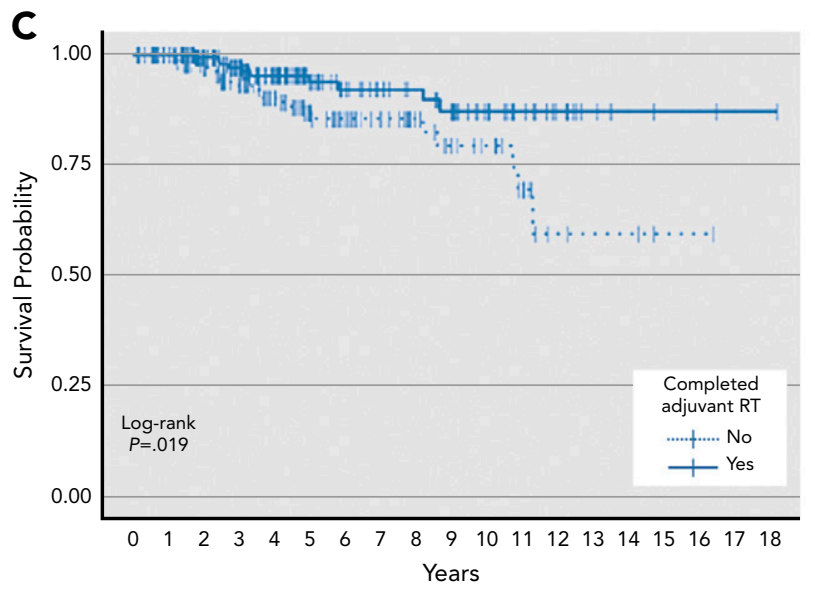

Number at risk

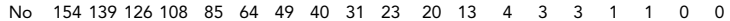

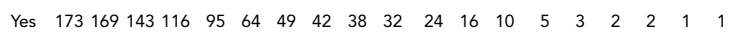

B

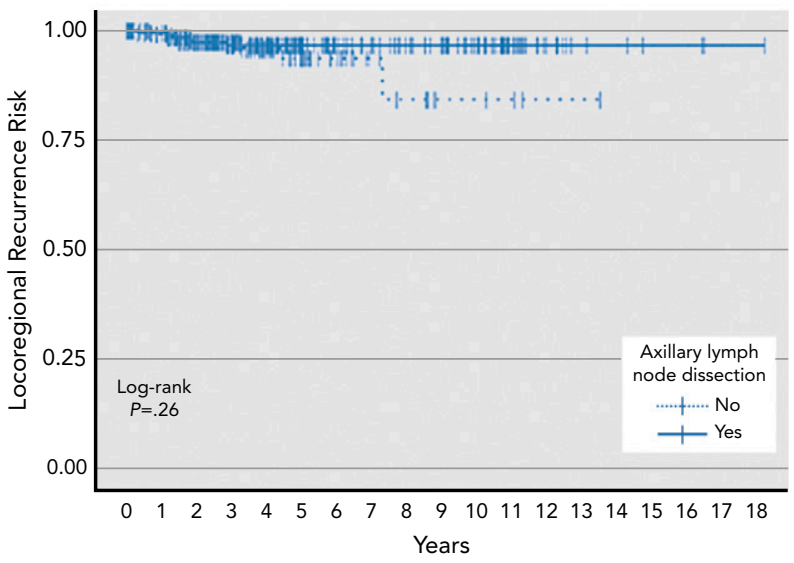

Number at risk

No $\begin{array}{lllllllllllllllllll}136 & 118 & 97 & 77 & 50 & 32 & 19 & 11 & 8 & 4 & 4 & 3 & 1 & 1 & 0 & 0 & 0 & 0 & 0\end{array}$

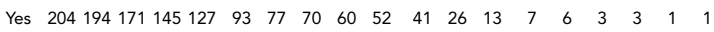

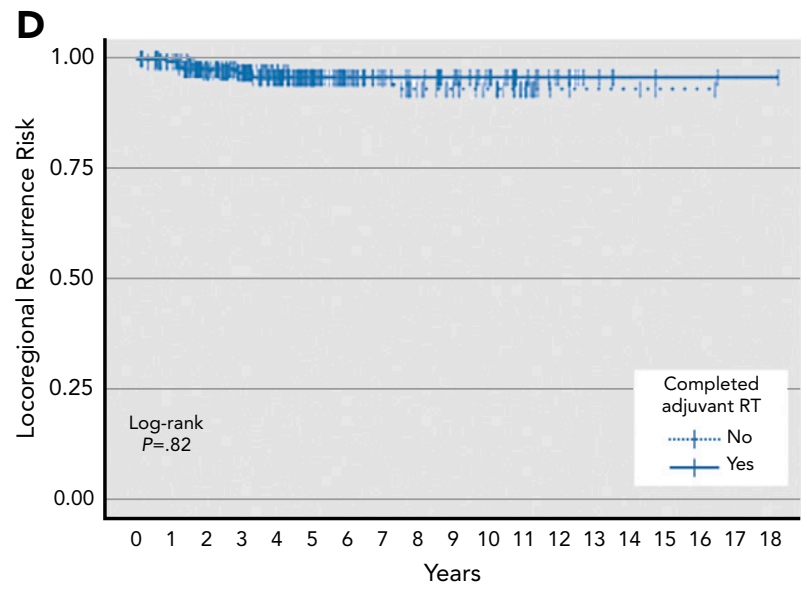

Number at risk

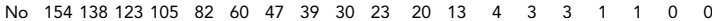
Yes $173 \begin{array}{llllllllllllllllll}168 & 140 & 114 & 92 & 63 & 48 & 41 & 37 & 32 & 24 & 16 & 10 & 5 & 3 & 2 & 2 & 1 & 1\end{array}$

Figure 2. Kaplan-Meier estimates of overall survival and locoregional recurrence by axillary lymph node dissection (A and B, respectively) and postmastectomy RT (C and D, respectively).

Abbreviation: RT, radiotherapy.

up to 2 axillary macrometastases. ${ }^{19}$ Patients will be randomized to adjuvant therapy alone versus standard of care, which consists of adjuvant therapy and axillary treatment (either CLND or axillary RT) with a primary endpoint of 5-year axillary recurrence. The key differences from ACOSOG Z0011 are inclusion of mastectomy patients, standardization of RT, and the inclusion of RT as axillary treatment. The study is expected to complete accrual in 2023. The SENOMAC trial (NCT02240472) is another prospective randomized trial addressing both mastectomy patients and another critique of ACOSOG Z0011: the omission of patients who received neoadjuvant therapy. ${ }^{20}$ Patients who have received neoadjuvant systemic therapy and either BCS or mastectomy will be eligible. The primary endpoint is 5-year breast cancer-specific survival. The study design allows management of patients based solely on SLNB staging to determine whether CLND is necessary.

All patients in our study were cN0; yet, after initial pathologic staging with SLNB, $44(22 \%)$ of 201 patients who underwent CLND had an increase in nodal stage. Previous studies have found that clinical examination of the axilla is relatively unreliable. A 1986 study reported a $39 \%$ erroneous examination rate of the axilla with a $45 \%$ false-negative and $29 \%$ false-positive rate. ${ }^{21} \mathrm{~A}$ more recent study reported approximately $33 \%$ false-negative and falsepositive rates. ${ }^{22}$ Therefore, it is not surprising to find patients in our cohort with a higher burden of axillary disease 


\begin{tabular}{|c|c|c|c|}
\hline Characteristic & No PMRT n (\%) & PMRT n (\%) & $P$ Value ${ }^{a}$ \\
\hline Total & $153(46.5)$ & $176(53.5)$ & \\
\hline $\begin{array}{l}\text { Median age at diagnosis } \\
\text { (IQR), y }\end{array}$ & $56(45-64)$ & $52(44-61)$ & .08 \\
\hline Median BMI (IQR), kg/m² & $25.9(22.9-29.7)$ & $26.4(22.9-30.7)$ & .54 \\
\hline Histology & & & .31 \\
\hline IDC & $118(78)$ & $123(69)$ & \\
\hline ILC & 19 (12) & $35(20)$ & \\
\hline IMC & $5(3)$ & $7(4)$ & \\
\hline Other & $11(7)$ & $11(7)$ & \\
\hline Tumor grade ${ }^{b}$ & & & .28 \\
\hline 1 & $21(14.5)$ & $17(10)$ & \\
\hline 2 & $65(42.5)$ & $88(50)$ & \\
\hline 3 & $66(43)$ & $69(40)$ & \\
\hline Pathologic T stage & & & $<.0001$ \\
\hline 1 & $87(56)$ & $57(31)$ & \\
\hline 2 & $64(43)$ & $95(52)$ & \\
\hline 3 & $2(1)$ & $24(17)$ & \\
\hline Overall stage & & & $<.001$ \\
\hline I & $37(24)$ & $7(4)$ & \\
\hline ॥ & $114(74.5)$ & $130(74)$ & \\
\hline III & $2(1.5)$ & $39(22)$ & \\
\hline Estrogen receptor & & & .04 \\
\hline Negative & $30(20)$ & $19(11)$ & \\
\hline Positive & $123(80)$ & $157(89)$ & \\
\hline Progesterone receptor & & & .04 \\
\hline Negative & $42(27.5)$ & $31(18)$ & \\
\hline Positive & 111 (72.5) & 145 (82) & \\
\hline
\end{tabular}

(continued)

than would be predicted by a negative clinical examination. Our institution has adopted the practice of evaluating the ipsilateral axilla with ultrasound in patients with clinical T2 or larger tumors; this practice has minimized understaging of the axilla based on clinical examination alone and is the subject of a separate investigation. ${ }^{23-25}$

We acknowledge the inherent limitations of retrospective studies. Our high rate of treatment with adjuvant chemotherapy may reflect differing practices after multidisciplinary tumor board discussion at our institution. This also highlights the importance of pathologic nodal status for clinical decisionmaking and treatment with adjuvant chemotherapy. We were also limited in our analysis by incomplete medical records and unavailability of certain data, most notably the details of adjuvant systemic therapy

\begin{tabular}{|c|c|c|c|}
\hline Characteristic & No PMRT n (\%) & PMRT n (\%) & P Value ${ }^{a}$ \\
\hline HER $2^{b}$ & & & .90 \\
\hline Negative & $134(88)$ & 157 (89) & \\
\hline Positive & $18(12)$ & $19(11)$ & \\
\hline $\begin{array}{l}\text { Median SLNs removed } \\
\text { (IQR), n }\end{array}$ & $3(2-4)$ & $3(2-4)$ & .33 \\
\hline $\begin{array}{l}\text { Median positive SLNs } \\
(\text { (IQR), n }\end{array}$ & $1(1-1)$ & $1(1-2)$ & .0003 \\
\hline CLND & & & 1.00 \\
\hline Yes & $93(61)$ & $108(61)$ & \\
\hline No & $60(39)$ & $68(39)$ & \\
\hline Extracapsular extension & & & .0002 \\
\hline Yes & $32(21)$ & $72(41)$ & \\
\hline No & $121(79)$ & $104(59)$ & \\
\hline \multicolumn{3}{|c|}{ Treated with adjuvant chemotherapy } & .0001 \\
\hline Yes & $98(64)$ & $147(83.5)$ & \\
\hline No & $55(36)$ & $29(16.5)$ & \\
\hline
\end{tabular}

Abbreviations: BMI, body mass index; CLND, completion axillary lymph node dissection; IDC, invasive ductal carcinoma; ILC, invasive lobular carcinoma; IMC, invasive mammary carcinoma; IQR, interquartile range; PMRT, postmastectomy radiation therapy; SLN, sentinel lymph node. aBold indicates statistically significant $P$ value.

${ }^{b}$ Data not available for all patients.

and RT. Therefore, we were unable to determine the contribution of other adjuvant therapies in the absence of CLND. We attempted to minimize selection bias by analyzing all mastectomy patients treated in the specified time frame, not just those who were similar to patients covered by ACOSOG Z0011 criteria; however, we acknowledge that there is inherent bias in selecting the patients who underwent CLND. These results should be evaluated cautiously due to the retrospective nature of the study. The results of ongoing prospective studies are eagerly awaited to better elucidate the best treatment of axillary disease and to validate the results of our study.

\section{Conclusions}

In female patients with cN0, SLN-positive invasive breast cancer who underwent mastectomy, CLND was not associated with differences in recurrence or OS, but it may impact the selection of patients who will benefit from adjuvant therapy. PMRT was also not associated with difference in recurrence but was associated with improved OS.

Submitted October 15, 2019; accepted for publication May 26, 2020.

Previous presentation: A portion of this work was presented at the 2019 Annual Cancer Symposium of the Society of Surgical Oncology; March 23-27, 2019; San Diego, California. Abstract 3074022. 
Author contributions: Study concept and design: J. Sun, Mathias, Laronga, W. Sun, Kiluk, Lee. Data collection: J. Sun, Mathias, W. Sun. Data analysis: Zhou, Fulp. Manuscript preparation: J. Sun, Lee. Critical revision: All authors.

Disclosures: The authors have disclosed that they have not received any financial consideration from any person or organization to support the preparation, analysis, results, or discussion of this article.
Funding: This work was supported in part by the Biostatistics and Bioinformatics Shared Resource at Moffitt Cancer Center, an NCl-designated Comprehensive Cancer Center (P30-CA076292).

Correspondence: M. Catherine Lee, MD, Department of Breast Oncology Moffitt Cancer Center, 10920 McKinley Drive, Tampa, FL 33612. Email: marie.lee@moffitt.org

\section{References}

1. Miller CL, Specht MC, Skolny MN, et al. Risk of lymphedema after mastectomy: potential benefit of applying ACOSOG Z0011 protocol to mastectomy patients. Breast Cancer Res Treat 2014;144:71-77.

2. Lucci A, McCall LM, Beitsch PD, et al. Surgical complications associated with sentinel lymph node dissection (SLND) plus axillary lymph node dissection compared with SLND alone in the American College of Surgeons Oncology Group trial Z0011. J Clin Oncol 2007;25:3657-3663.

3. Giuliano $A E, M c C a l l L$, Beitsch $P$, et al. Locoregional recurrence after sentinel lymph node dissection with or without axillary dissection in patients with sentinel lymph node metastases: the American College of Surgeons Oncology Group Z0011 randomized trial. Ann Surg 2010;252: 426-432, discussion 432-433.

4. Galimberti V, Cole BF, Zurrida S, et al. Axillary dissection versus no axillary dissection in patients with sentinel-node micrometastases (IBCSG 23-01): a phase 3 randomised controlled trial. Lancet Oncol 2013;14: 297-305.

5. Giuliano AE, Ballman K, McCall L, et al. Locoregional recurrence after sentinel lymph node dissection with or without axillary dissection in patients with sentinel lymph node metastases: long-term follow-up from the American College of Surgeons Oncology Group (Alliance) ACOSOG Z0011 randomized trial. Ann Surg 2016;264:413-420.

6. Giuliano AE, Ballman KV, McCall L, et al. Effect of axillary dissection vs no axillary dissection on 10-year overall survival among women with invasive breast cancer and sentinel node metastasis: the ACOSOG Z0011 (Alliance) randomized clinical trial. JAMA 2017;318:918-926.

7. Galimberti V, Cole BF, Viale G, et al. Axillary dissection versus no axillary dissection in patients with breast cancer and sentinel-node micrometastases (IBCSG 23-01): 10-year follow-up of a randomised, controlled phase 3 trial. Lancet Oncol 2018;19:1385-1393.

8. Donker M, van Tienhoven G, Straver ME, et al. Radiotherapy or surgery of the axilla after a positive sentinel node in breast cancer (EORTC 1098122023 AMAROS): a randomised, multicentre, open-label, phase 3 noninferiority trial. Lancet Oncol 2014;15:1303-1310.

9. Rutgers $\mathrm{E}$, Donker M, Poncet $\mathrm{C}$, et al. Radiotherapy or surgery of the axilla after a positive sentinel node in breast cancer patients: 10 year follow up results of the EORTC AMAROS trial (EORTC 10981/22023) [abstract] Cancer Res 2019;79(4 Suppl):Abstract GS4-01.

10. Gradishar WJ, Anderson BO, Abraham J, et al. NCCN Clinical Practice Guidelines in Oncology: Breast Cancer. Version 6.2020. Accessed August 25,2020 . To view the most recent version, visit NCCN.org

11. American Society of Breast Surgeons. Consensus guideline on the management of the axilla in patients with invasive/in-situ breast cancer. Accessed August 25, 2020. Available at: https://www.breastsurgeons.org/ docs/statements/Consensus-Guideline-on-the-Management-of-the-Axilla.pdf?v2
12. Giuliano $A E$, Hunt $K K$, Ballman $K V$, et al. Axillary dissection vs no axillary dissection in women with invasive breast cancer and sentinel node metastasis: a randomized clinical trial. JAMA 2011;305:569-575.

13. McGale P, Taylor C, Correa C, et al. Effect of radiotherapy after mastectomy and axillary surgery on 10-year recurrence and 20-year breast cancer mortality: meta-analysis of individual patient data for 8135 women in 22 randomised trials. Lancet 2014;383:2127-2135.

14. Recht A, Comen EA, Fine RE, et al. Postmastectomy radiotherapy: an American Society of Clinical Oncology, American Society for Radiation Oncology, and Society of Surgical Oncology focused guideline update. Pract Radiat Oncol 2016;6:e219-234.

15. Milgrom S, Cody H, Tan L, et al. Characteristics and outcomes of sentinel node-positive breast cancer patients after total mastectomy without axillary-specific treatment. Ann Surg Oncol 2012;19:3762-3770.

16. FitzSullivan E, Bassett RL, Kuerer HM, et al. Outcomes of sentinel lymph node-positive breast cancer patients treated with mastectomy without axillary therapy. Ann Surg Oncol 2017;24:652-659.

17. Snow R, Reyna C, Johns C, et al. Outcomes with and without axillary node dissection for node-positive lumpectomy and mastectomy patients. Am J Surg 2015;210:685-693

18. Joo JH, Kim SS, Son BH, et al. Axillary lymph node dissection does not improve post-mastectomy overall or disease-free survival among breast cancer patients with 1-3 positive nodes. Cancer Res Treat 2019;51: 1011-1021.

19. Goyal A, Dodwell D. POSNOC: a randomised trial looking at axillary treatment in women with one or two sentinel nodes with macrometastases. Clin Oncol (R Coll Radiol) 2015;27:692-695.

20. de Boniface J, Frisell J, Andersson Y, et al. Survival and axillary recurrence following sentinel node-positive breast cancer without completion axillary lymph node dissection: the randomized controlled SENOMAC trial. BMC Cancer 2017;17:379.

21. Sacre RA. Clinical evaluation of axillar lymph nodes compared to surgical and pathological findings. Eur J Surg Oncol 1986;12:169-173.

22. Specht MC, Fey JV, Borgen PI, et al. Is the clinically positive axilla in breast cancer really a contraindication to sentinel lymph node biopsy? J Am Coll Surg 2005;200:10-14.

23. Joh JE, Han G, Kiluk JV, et al. Indications for axillary ultrasound use in breast cancer patients. Clin Breast Cancer 2012;12:433-437.

24. Lee MC, Eatrides J, Chau A, et al. Consequences of axillary ultrasound in patients with T2 or greater invasive breast cancers. Ann Surg Oncol 2011; 18:72-77.

25. Turaga KK, Chau A, Eatrides JM, et al. Selective application of routine preoperative axillary ultrasonography reduces costs for invasive breast cancers. Oncologist 2011;16:942-948.

\section{See JNCCN.org for supplemental online content.}


Supplemental online content for:

\section{Impact of Axillary Dissection Among Patients With Sentinel Node-Positive Breast Cancer Undergoing Mastectomy}

James Sun, MD; Brittany J. Mathias, MD; Christine Laronga, MD; Weihong Sun, MD; Jun-Min Zhou, PhD; William J. Fulp, MS; John V. Kiluk, MD; and M. Catherine Lee, MD

J Natl Compr Canc Netw 2021;19(1):40-47

eTable 1: Clinicopathologic Characteristics of Patients With Estrogen Receptor-Positive Disease Compared by Complete Lymph Node Dissection 


\section{eTable 1. Clinicopathologic Characteristics of Patients With ER-Positive Disease Compared by CLND}

\section{Characteristic}

Total

Median age at diagnosis (IQR), y

Median BMI (IQR), $\mathrm{kg} / \mathrm{m}^{2}$

Median tumor size (IQR), cm

Histology

IDC

IMC

Other

Tumor grade

$\frac{1}{2}$

3

Pathologic T stage

1

2

3

Overall stage

II

No CLND

n (\%)

118 (42)

$56.5(47-64)$

$25.9(23.3-30.1)$

$2.1(1.5-3)$

84 (71)

$23(18.5)$

$3(2.5)$

$8(7)$

$18(15)$

67 (57)

$33(28)$

$56(47)$

$54(46)$

8 (7)

28 (24)

$82(69)$

$8(7)$

Lymphovascular invasion

\begin{tabular}{lr} 
Yes & $36(31)$ \\
\hline No & $80(68)$ \\
\hline Indeterminate & $2(1)$
\end{tabular}

Progesterone receptor

Negative

Positive

HER $2^{\text {b }}$

Negative

Positive

Extracapsular extension

Yes

No

Surgery timing ${ }^{c}$

Before September 2010

After September 2010

Treated with adjuvant RT

Yes

No

$110(93)$

$110(94)$

7 (6)

90 (76)

11 (9)

107 (91)

54 (46)

\section{CLND}

n (\%)

P Value ${ }^{a}$

$162(58)$

52.5 (44-63)

$26.8(23.1-30.2)$

$2.5(1.63-3.5)$

.02

.90

.05

.80

111 (69)

31 (19)

8 (5)

$12(7)$

.23

19 (12)

$81(51)$

60 (37)

.49

69 (43)

$76(47)$

$17(10)$

$10(6)$

$121(75)$

31 (19)

71 (44)

$87(54)$

4 (2)

$20(12)$

$142(88)$

14 (9)

$28(24) \quad 70(43)$

$92(57)$

$92(57)$

70 (43)

$64(54) \quad 93(57)$
.05

.18

$<.0001$
$69(43)$ 


\begin{tabular}{|c|c|c|c|}
\hline Characteristic & $\begin{array}{c}\text { No CLND } \\
\text { n (\%) }\end{array}$ & $\begin{array}{l}\text { CLND } \\
\mathrm{n}(\%)\end{array}$ & P Value ${ }^{a}$ \\
\hline Treated with adjuvant chemotherapy & & & $<.0001$ \\
\hline Yes & $62(53)$ & $135(83)$ & \\
\hline No & $56(47)$ & 27 (17) & \\
\hline
\end{tabular}

Abbreviations: BMI, body mass index; CLND, completion axillary lymph node dissection; ER, estrogen receptor; IDC, invasive ductal carcinoma; ILC, invasive lobula carcinoma; IMC, invasive mammary carcinoma; IQR, interquartile range; $R T$, radiotherapy.

aBold indicates statistically significant $P$ value.

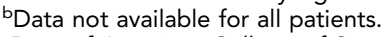

cDate of American College of Surgeons Oncology Group Z0011 publication. 